\title{
The Design of Navigation System Detector for a Certain Self-propelled Anti-aircraft Gun
}

\author{
Yi Huang ${ }^{1, a}$, Xiusheng Duan ${ }^{2, b}$, Hailong Zhang ${ }^{3, c}$, Yingjie Dai ${ }^{4, d}$ \\ ${ }^{1,2}$ Department of Electrical Optical and Engineering, Ordnance Engineering College, Shijiazhuang, \\ 050003, China \\ ${ }^{3}$ China Xi'an Satellite Control Center, Xi'an, 710043, China \\ ${ }^{4}$ The Fifth Department, Air Defense Forces Academy, Zhengzhou, 450052, China \\ aemail: \\ dd_huangyi@163.com, bemail:sjzdxsh@163.com, cemail:wosihailong@sina.com, ${ }^{d}$ email:dyj105.cz \\ zx@163.com
}

Keywords: Navigation system detector; fault diagnosis; performance testing

\begin{abstract}
Navigation system for self-propelled anti-aircraft gun is to provide location, course and vehicle posture angle to support independent operation and good shooting ability while vehicle moving. So its overall performance testing and fault diagnosis is crucial important. In this paper, based on some related technologies, the design of navigation system detector for self-propelled anti-aircraft gun is completed. The navigation system detector we designed has reasonable structure, practical function, convenient maintenance and safe usage, and has been widely equipped in army. According to the feedback, the effect of application of this detector is good.
\end{abstract}

\section{Introduction}

To implement the function of independent operation and shooting ability when moving, as well as synergy among the vehicles for Self-propelled Anti-aircraft Gun, vehicle navigation system is needed, which can provide location, course and vehicle posture angle. The navigation system is mainly composed of the azimuth vertical reference instrument, the fast automatic north seeking device and the vehicle body information processor, etc[1]. The navigation system detector can detect the overall performance and the main components of the navigation system. It's easy to find fault timely to ensure the good technical state of the navigation system.

\section{Composition and function of the navigation system detector}

Composition of the navigation system detector. The System adopts split structure, and mainly composed of the main control computer (simply, the master), the front testing computer (simply, the testing computer), communication control box and connecting cables, test probes, etc[4].

The master adopts laptop, and communicates with the testing computer through the serial $\mathrm{C} / \mathrm{S}$ network. It sends out a test instruction based on the operation of a test personnel, and receives the test data and preliminary analysis results when the test is complete, and then carries out further feature extraction, data timing analysis, etc.

The testing computer is mainly composed of industrial personal computer(IPC)、PCI-1802L multi-function data acquisition card, PCI-7296 digital I/O card, data pre-processing board, control box elevation board v probes etc.. In the procedure of test on monomers, the testing computer is connected with the monomers through testing cable. The signal of each pin is collected under the control of the master, and the data is analyzed preliminary, then uploaded to the master.

The communication control box mainly complete the wired communication between the master and the testing computer, meanwhile it can indicate the working state of the testing computer[2].

Main functions of the navigation system detector. On the basis of the working principle of the system, a system to board level fault tree of the navigation system detector and fault diagnosis expert database are established. Main functions include automatic test of signal among monomers 
of the navigation system, pen test of circuit board inside the monomers and fault diagnosis.

\section{Overall system design}

Overall system structure. The determination of the overall framework of the system is based on the background of actual equipment, the needs of the military technical, and the guiding ideology of easy using, the principle of structure miniaturization, detection automation, and rich in resources, easy to operate. The hardware platform of the detection and diagnosis system is composed of a portable cabinet, industrial control board, a notebook computer (or a desktop computer). The system test resources are composed of PCI-7296 and PCI-1802L card. The state signal of the equipment is introduced by signal conditioning circuit which includes power supply circuit, power amplifier circuit, Scott transformer, signal voltage dividing circuit, digital I/O input and output circuit etc[3].

Software design considers the fault detection system, expert diagnosis system and equipment self-checking function. According to the signal characteristics of the cables among monomers, the software design adopts flexible fault detection methods, under the premise of the fault coverage and diagnostic accuracy.

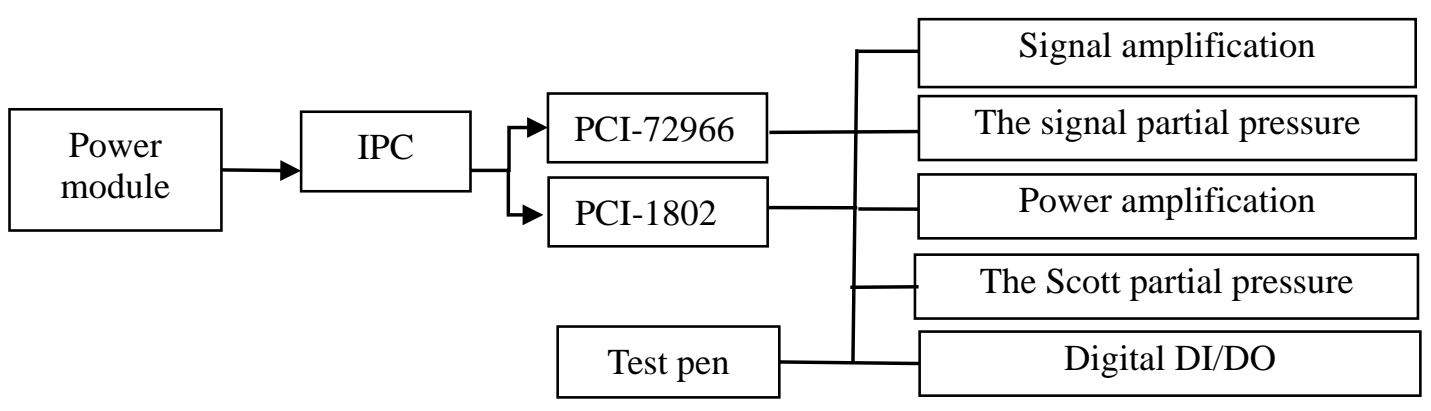

Fig.1. Hardware system structure diagram

Hardware system design. Because of the narrow space of anti-aircraft, it is difficult to carry out the internal test. At the same time, in order to meet the test requirements in working conditions of self-propelled antiaircraft, the hardware adopts split structure. The hardware can complete the data acquisition task under the control of the master, and preliminary analysis data. Then the analysis results are transmitted to the master through the data transmission module. All tests are done through the master by operator. The master is notebook computer, communicating with testing computer through the serial C/S network. It sends out a test instruction based on the operation of a test personnel, receives the test data and preliminary analysis results when the test is complete, and then carries out further feature extraction, data timing analysis, display function, etc[5].

Hardware design mainly includes two parts, the design of the testing computer and the design of the communication control box.

Testing computer designs based on IPC, three function cards are fixed in the form of PCI bus in the industrial board, the overall structure schematic as shown in the figure 1.

Software design. Detection and diagnosis system software of Navigation system developments mainly using Borland $\mathrm{C}++$ Builder 6.0, the main software module and its function as shown in Table1. 
Table 1 Main software module and its function for navigation system detector

\begin{tabular}{|c|c|c|}
\hline Number & Name & Main function \\
\hline 1 & $\begin{array}{l}\text { The acquisition and processing module } \\
\text { of weak signal }\end{array}$ & $\begin{array}{l}\text { Used for testing the weak signal which input or } \\
\text { output by inertial component. }\end{array}$ \\
\hline 2 & The gathering module of analog signals & $\begin{array}{l}\text { Used for sampling the analog signals like voltage } \\
\text { (current) }\end{array}$ \\
\hline 3 & The gathering module of digital signals & $\begin{array}{l}\text { Used for sampling signal for the parallel } \\
\text { communication、 sequential control and status. }\end{array}$ \\
\hline 4 & The adapter module of signal interface & $\begin{array}{l}\text { Used for pretreatment for analog signals and digital } \\
\text { signals like gain control, filter, switch for the signal } \\
\text { and channel, etc. }\end{array}$ \\
\hline 5 & $\begin{array}{l}\text { The measuring module of shaft angle } \\
\text { signal }\end{array}$ & Used for testing the course angle \\
\hline 6 & The control module of communication & $\begin{array}{l}\text { Used for controlling the command and data } \\
\text { transmission of the main control computer and test } \\
\text { computer }\end{array}$ \\
\hline 7 & $\begin{array}{l}\text { The module of signature analysis and } \\
\text { extraction }\end{array}$ & $\begin{array}{l}\text { Used for analyzing and processing signals and } \\
\text { extracting its feature information }\end{array}$ \\
\hline 8 & The module of fault diagnosis & $\begin{array}{l}\text { Realized the fault diagnostic reasoning and } \\
\text { location based on testing }\end{array}$ \\
\hline 9 & $\begin{array}{l}\text { The module of human-computer } \\
\text { interface }\end{array}$ & Used for operation control and display \\
\hline
\end{tabular}

\section{Main technology and the accomplishment}

The design of the digital signal acquisition module. In the system, test hardware selection, signal acquisition mode and process control are carried on in accordance with the feature of test signal. According to the difference of signal characteristics, the system designs different acquisition mode in order to acquire the best acquisition signal and assure the test accuracy and configuration flexibility.

(1)Continuous acquisition mode

When the length and the time of acquisition can be confirmed in advance, you can acquire the fixed-length data by setting the number of data collected. When in cases such as observing the entire work sequence or the condition of interface signal and judging signal characteristics qualitatively, you can achieve continuous data acquisition by using the double buffer area DMA principle.

(2)Trigger mode

A main problem in acquiring data by trigger mode is the selection of trigger signal. Normally, the work sequence of system is approximately fixed, you can select trigger signal by analyzing the sequence. If the interval of trigger signal and tested signal is too long, then you can achieve it by inserting the delay circuit. When in fault condition, the system sequence might be changed, some of the signal become irregular or nonexistence. If trigger signal lost, the acquisition system can be caused to a drop-dead halt, waiting for the trigger signal, and the subsequent test cannot go on either. Therefore, only when insert obligatory trigger signal can lead the acquisition system back to the normal condition.

In order to avoid system halting which happens when the trigger signal is lost during the testing, the system uses continuous fixed length acquisition at first and judges whether the start control signal of interface signal exists or not, and the system enters into the trigger acquisition module only if these signals are normal. Of course, because of the stringency of the time sequence, it is impossible to judge every period, so the judgment only happens at the beginning of every test and also the system sets up signs to permit triggering or not to allow trigger.

The Design of conditioning circuit for analog signals. Every signal comes from different tested cables and it is impossible for detecting instrument to design appropriative conditioning circuit for every stitch, so for similar signals, it needs to use commutation circuit to make time division multiplexing of conditioning circuit come true. 
According to the input range of analog switch chip, sampled signals are divided into three parts according to peak amplitude. The signals in $[-12 \mathrm{~V},+12 \mathrm{~V}]$ are direct signals, the signals in $[-100 \mathrm{~V}$, $+100 \mathrm{~V}]$ are strong signals and others are partial pressure signals. The particular circuit principle is as shown in figure 2 .

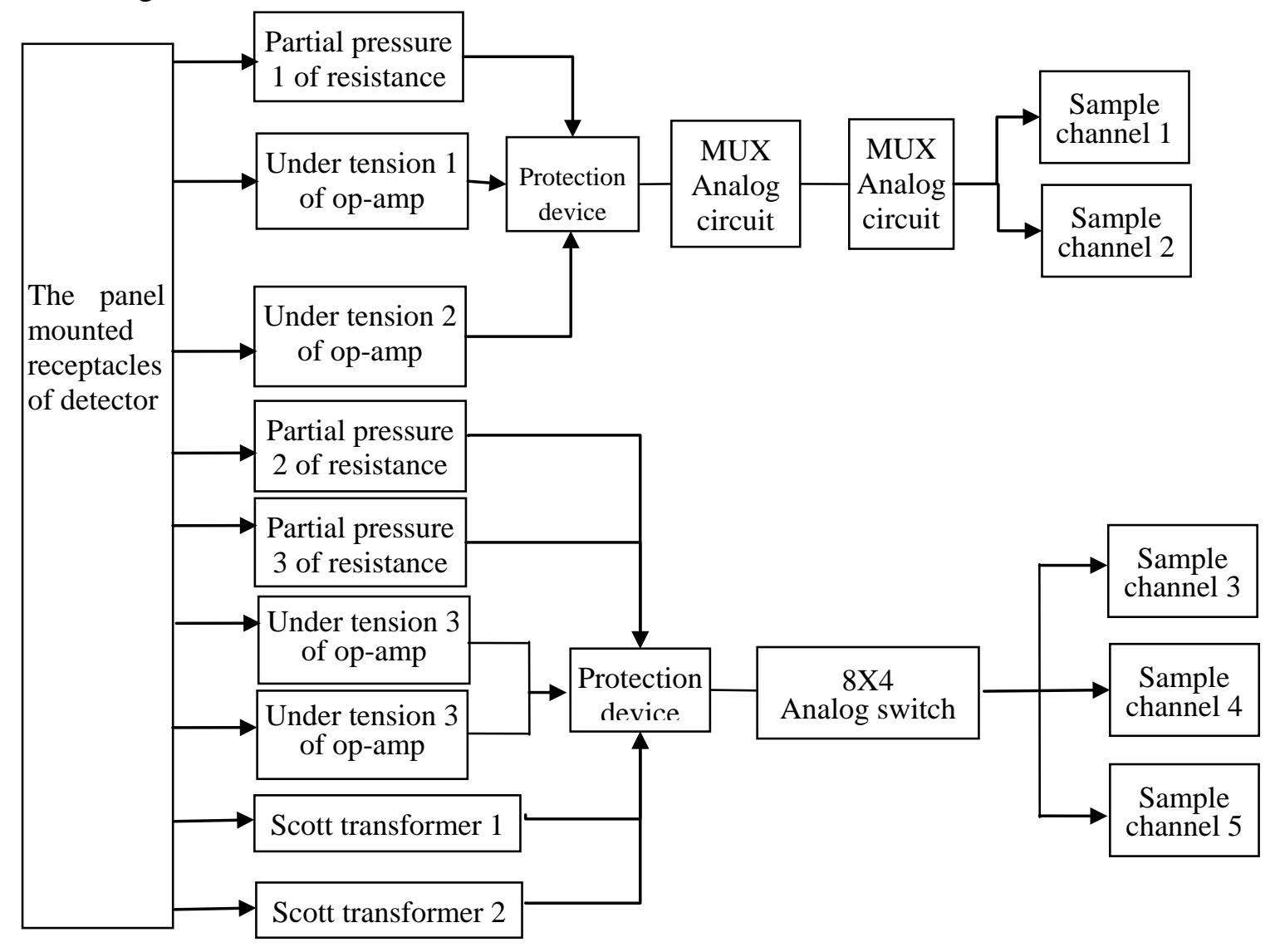

Fig.2. The structure of conditioning circuit of analog signals

The direct signal can further be divided into the power signal and the control signal. In specific equipment test, the first matter of power signal is whether it exists, and the next is the smooth degree of signal. The current is not tested generally. Control signal need testing many performance parameters. When our detector deals with these direct signals, it adopts flexible analysis methods, namely it mainly contrasts the peak value, the peak-peak value, the duty cycle for power signal. For control signal, some indexes such as the frequency, the type of signals, the signal polarity and the maximum ascendant time would be tested on the foundation of three indexes mentioned above. There are two special points, one of which is for partial signals point by point comparison method is actualized in special time such as in system boot process. Namely locating the signal in the state signal of the whole process to find the standard signal in special time and making judgments in a range of fault tolerance. For example, the gyro compensation, coordination, stability and other signals for navigation system in system boot process, not only have the requirements of frequency, amplitude and other signal characteristics, but also have strict requirements of time sequence and time interval. By comparing with the normal signal in the normal state, the detection of these special signals can be effectively accomplished.

Because some signals among control signals have time sequence relations, our system has also done the related design. Under the control of the same sampling clock, the parallel channel is used to collect these signals, and the corresponding algorithms are used to analyze their temporal relation.

Strong signals generally contain power signals and motor control signals, whose basic method can be referred to in the direct signal part. Specially, aiming at the feature that test object has lots of $400 \mathrm{~Hz}$ medium frequency signals, combined with hardware acquisition system of indicators, our system designed new frequency solving algorithm. Through many experiments, we select the 
appropriate sampling time, sampling frequency and sampling points, efficiently improve the calculating accuracy of medium frequency signals, and calculating accuracy of $115 \mathrm{~V}$ signals between $300 \mathrm{~Hz}$ to $500 \mathrm{~Hz}$ drop to $1 \%$.

Partial pressure signals can also be divided into power signals and control signals, at the same time, their dividing circuit differs. The former uses precision resistance voltage divider circuit, which simplifies the circuit under the premise of detection precision. The latter uses a feedback circuit constituted by stable gaining amplifier to realize signals divide, and it can ensure the integrity of the signal characteristics under the premise of satisfy extent.

The procedure design of fault diagnosis. Diagnostic strategy is based on fault diagnosis knowledge model, finds fault location by cycle of testing and judgement, and resolve multiple faults diagnose. The inference engine is the related procedures to make diagnostic procedures required by the diagnostic strategy carry out automatically.

Inference engine should be made up of two parts, thanks to knowledge base dividing into experience knowledge and theory knowledge two parts. First diagnose with experience knowledge. if it can be done fast, then diagnosis is finished. If experience knowledge is not perfect and fail to find failure location, turn to theory knowledge. Reasoning diagram is as shown in the figure 3.

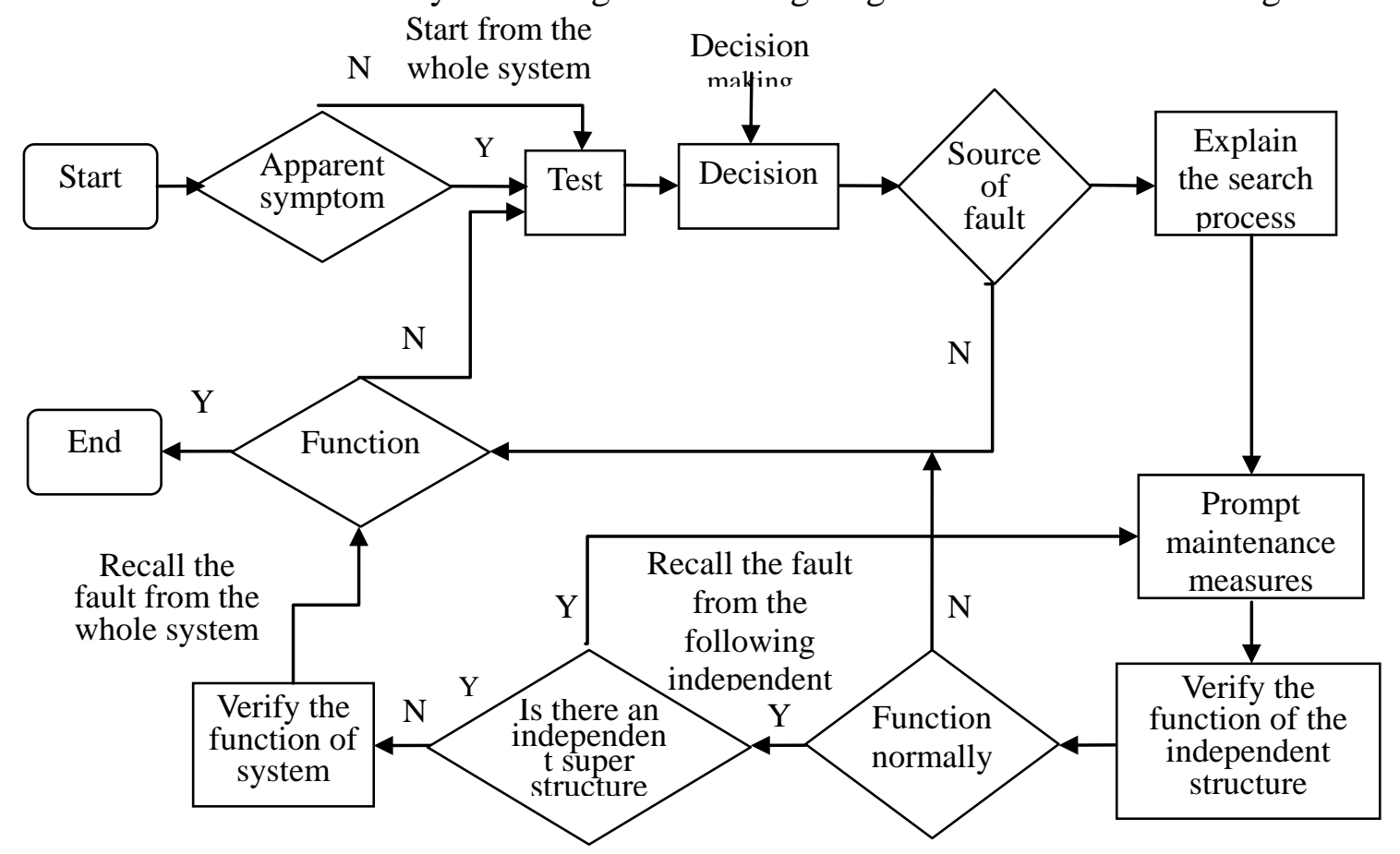

Fig.3. The reasoning diagram of fault diagnosis

\section{Conclusion}

This paper is closely connected with the actual demand of navigation system for anti-aircraft gun. Based on some related technologies, the design of navigation system detector for self-propelled anti-aircraft gun is completed. The navigation system detector we designed has reasonable structure, practical function, convenient maintenance and safe usage, and has been widely equipped in army. According to the feedback, the effect of application of this detector is good.

\section{References}

[1]Shen Peng, Hongming Zhang, Dacheng Liu. The Research of Fault Inspection Based on Integrated Navigation System[J]. Journal of Aircraft Measurement and Control. 2009, (3).

[2] Hong Jin, Hong Zhang. A Simple Method for Fault Detection of Navigation System[J].The 
Delivery Technology of Missile and Spaceflight.1998, (1).

[3] Lingxia Zhang. The Research of Navigation System's Fault Inspection and diagnosis and Some Related Theoretical Problems[D]. Harbin Northwestern Polytechnical University. 2006.

[4] Xuecong Li. The Research of Fault Inspection and diagnosis Based on Integrated Navigation System[D]. Harbin Engineering University. 2005.

[5]Guiqiu SONG, Yue PAN. The Research of Intelligent Vehicles Aided Navigation System Based on Machine Vision[C]. Proceedings of the International Conference on Mechanical Engineering and Mechanics. 2007. 